\title{
Factors for Active Site Generation and Pore Development in Fuel Cell Catalysts Formed from Glucose/Nitrogen Source/Fe Salts
}

\author{
Jun Maruyama, ${ }^{\text {a,* }}$ Nobutaka Fukui, ${ }^{\text {b }}$ Masayuki Kawaguchi, ${ }^{\text {, }}$ Takahiro Hasegawa, ${ }^{\text {a }}$ \\ Hiroaki KawAno, ${ }^{\mathrm{a}}$ Tomoko FuKUhara, ${ }^{\mathrm{a}}$ and Satoshi IwASAKI ${ }^{\mathrm{a}}$
a Environmental Technology Research Division, Osaka Municipal Technical Research Institute (1-6-50 Morinomi- ya, Joto-ku, Osaka 536-8553, Japan)
${ }^{\mathrm{b}}$ Graduate School of Engineering, Osaka Electro-Communication University (18-8 Hatsu-cho, Neyagawa, Osaka \\ 572-8530, Japan)
}

Received October 4, 2010 ; Accepted November 30, 2010

\begin{abstract}
A carbon-based noble-metal-free fuel cell cathode catalyst was formed by heat treatment of a mixture of glucose, Fe gluconate, and 1,4-diamino-2,3-dicyano-9,10-anthraquinone as a nitrogen source. The factors for the formation of the active site and the pore development were the heat-treatment temperature and the content of the nitrogen source in the starting mixture. The activity for oxygen reduction was dependent on the active site and the pore development, which were optimized to form the catalyst with the improved activity compared to those formed from various glucose/nitrogen source/Fe salts mixtures
\end{abstract}

Key Words : Noble-metal-free Catalyst, Carbon Material, Active Site Formation, Pore Development

\section{Introduction}

World-wide dissemination of polymer electrolyte fuel cells (PEFCs) in increasing vehicles and domestic electricity-heat generators needs noble-metal-free electrode catalysts, which are free from the problems of the conventional Pt-based catalyst: the resource limitation and the cost inflation.

One of the noble-metal-free catalysts is a porous carbon material with $\mathrm{Fe}$ coordinated by $\mathrm{N}$ atoms $\left(\mathrm{Fe}-\mathrm{N}_{x}\right.$ moiety) embedded in the pore surface functioning as the active site for the cathodic $\mathrm{O}_{2}$ reduction. The promising performance was recently demonstrated; ${ }^{1)}$ nevertheless, the sufficient activity and durability have not attained yet. Thus, it is currently important to obtain the information and attempt their improvement.

We have shown that the formation of the porous carbon material with the $\mathrm{Fe}-\mathrm{N}_{x}$ moiety was possible by the heat treatment of the mixture of Fe salts, glucose, nitrogen-containing compounds, such as amino acids, purine bases, pyrimidine bases. ${ }^{2-4)}$ The porous carbon matrix was derived from glucose because these nitrogen sources decompose during the heat treatment. The increase in the number of nitrogen atoms in the molecule led to the efficient generation of the active site. However, the detailed mechanism of the pore development and the active site formation is unclear at present.

We recently found that the carbon material with the $\mathrm{Fe}-\mathrm{N}_{x}$ moiety was formed from $\mathrm{Fe}$ gluconate and 1,4-diamino-2,3-dicyano-9,10-anthraquinone (DADCAQ) without glucose, although the inclusion of magnesium oxide inside the carbon material and its removal was necessary to develop the pores inside the carbon material. ${ }^{5}$ DADCAQ was chosen as the nitrogen source because it is a raw material of phthalocyanine $(\mathrm{Pc})$ synthesis and the efficient generation of the $\mathrm{Fe}-\mathrm{N}_{x}$ moiety was expect- ed.

In this study, we attempted the formation of the carbon material with the $\mathrm{Fe}-\mathrm{N}_{x}$ moiety by using DADCAQ and glucose in order that both the pore development and the active site generation occurred efficiently, and that the information on the activity improvement was obtained through forming it in various conditions.

\section{Experimental}

\subsection{Formation of carbon material}

Glucose, DADCAQ, and iron(II) gluconate dihydrate were mixed and then finely ground. The molar ratios of the DADCAQ to glucose were $0.25,0.5$, and 1 and the Fe content in the mixture was $1 \mathrm{wt} \%$. The mixture was heated at $150^{\circ} \mathrm{C}$ in air for $24 \mathrm{~h}$ for dehydration of glucose, which is often carried out before the carbonization of glucose. After grinding this dehydrated mixture similarly, the powder was heated in $100 \mathrm{~cm}^{3} \mathrm{~min}^{-1}$ of flowing Ar at $700,800,900$, and $1000^{\circ} \mathrm{C}$ for $2 \mathrm{~h}$ after raising the temperature at $5{ }^{\circ} \mathrm{C} \mathrm{min}^{-1}$. The sample was finely ground again and a treatment with an acid solution was carried out to remove soluble $\mathrm{Fe}$ species. The treatment was performed in $0.5 \mathrm{M} \mathrm{H}_{2} \mathrm{SO}_{4}$ at a boiling temperature for 1 h, followed by filtering, washing with high-purity water, and drying in vacuum at room temperature. For convenience, the carbon material formed from the mixture in which the DADCAQ/glucose molar ratio was 0.25 at the heat-treatment temperature of $1000^{\circ} \mathrm{C}$ is hereafter called G1AQ0.25Fe1000. All the others used the similar abbreviations.

\subsection{Characterization of the carbon materials}

The adsorption isotherm of $\mathrm{N}_{2}$ onto the carbon material was measured using an automatic $\mathrm{N}_{2}$ adsorption apparatus (Belsorp 28, Nippon Bell) at $-196^{\circ} \mathrm{C}$. The specific surface area was determined by the Brunauer-Emmet- 
Teller (BET) plot of the isotherm, and the pore volume by the amount of adsorbed $\mathrm{N}_{2}$ at the relative pressure of 0.931 . The mean pore diameter was calculated assuming the pores to be cylindrical and using the equation

$$
d=4 V_{\mathrm{p}} / S
$$

where $d$ is the mean pore diameter, $V_{\mathrm{p}}$ is the pore volume, and $S$ is the specific surface area. The measurements of the extended X-ray absorption fine structures (EXAFS) at the Fe K-edge were performed in the transmission mode in air at room temperature using synchrotron radiation at the beam line of BL14B2 of SPring-8 at the Japan Synchrotron Radiation Research Institute. A fourier transformation was performed on a $k^{3}$-weighted EXAFS spectrum using the REX2000 program (Rigaku) to calculate the pseudo-radial distribution function (RDF).

\subsection{Evaluation of catalytic activity}

The activity of the catalyst was evaluated by fixing it on the surface of a rotating glassy carbon disk electrode (GC RDE) as a catalyst layer and immersing it in $0.1 \mathrm{M}$ $\mathrm{HClO}_{4}$. An aliquot of $10 \mathrm{mg}$ of the finely ground catalyst and $1 \mathrm{mg}$ of carbon black (Vulcan XC-72R, Cabot) as the electron-conductive agent were added to $1 \mathrm{~cm}^{3}$ of the 5 wt\% Nafion solution. The mixture was ultrasonically dispersed to produce a catalyst paste. A GC RDE (BAS), which consisted of a GC rod sealed in a Kel-F holder, was polished with 2000 grit emery paper (Sumitomo 3M) and then ultrasonically cleaned in high-purity water for use as a support for the catalyst layer. The geometric surface area of the electrode was $0.071 \mathrm{~cm}^{2}$ (diameter, 3 $\mathrm{mm}$ ). A $1 \mathrm{~mm}^{3}$ volume of the paste was pipetted onto the GC surface, and to shield it from the irregular air stream generated by a ventilator, the electrode was immediately placed under a glass cover until the layer was formed. This operation was repeated once again to load $20 \mu \mathrm{g}$ of the carbon material on the GC surface. After removal of the glass cover, the layer was further dried overnight at room temperature.

An electrochemical analyzer (100B/W, BAS) and an RDE glass cell were used for the electrochemical measurements. The counter electrode was a Pt wire, and the reference electrode was a reversible hydrogen electrode (RHE). All potentials were referred to the RHE. Cyclic voltammograms for the catalyst layers were recorded in Ar-saturated $0.1 \mathrm{M} \mathrm{HClO}_{4}$ at $25^{\circ} \mathrm{C}$. The potential was scanned between 0.05 and $1.3 \mathrm{~V}$ at a scan rate of $50 \mathrm{mV}$ $\mathrm{s}^{-1}$. The current-potential relationships were obtained in an $\mathrm{O}_{2}$-saturated $0.1 \mathrm{M} \mathrm{HClO}_{4}$ at $25^{\circ} \mathrm{C}$ and various rotation speeds. The scan rate of the potential was fixed at 10 $\mathrm{mV} \mathrm{s}^{-1}$. The background current was similarly measured in an Ar atmosphere without rotation.

\section{Results and Discussion}

\subsection{Factors for pore development}

Table 1 shows the yields for the carbon material, the specific surface area, and the mean pore diameter. The yield showed the downward tendency with increases in the heat-treatment temperature and the glucose content in the starting mixture. The increases in these two parameters caused the increases in the specific surface area and the mean pore diameter with exceptions of the extraordinary large mean pore diameter of G1AQ0.5Fe700 and the smaller values of G1AQ0.5Fe1000 than those of G1AQ0.5Fe900. The pore began to develop above $800^{\circ} \mathrm{C}$ and G1AQ0.5Fe700 was different from the other samples in regard to the pore structure. The smaller specific surface area and the mean pore diameter in G1AQ0.5Fe1000 than in G1AQ0.5Fe900 was due to the pore shrinkage, which is often observed in carbon materials formed in the high temperature. Nevertheless, the results indicated that the high heat-treatment temperature was necessary for the pore development and that the low DADCAQ content was also advantageous for it.

\subsection{Factors for active site generation}

The generation of the $\mathrm{Fe}-\mathrm{N}_{x}$ moiety was investigated by EXAFS. Figure 1 shows the RDFs for the carbon materials, $\mathrm{Fe}$ foil, and $\mathrm{FePc}$. In the RDF for $\mathrm{FePc}$, the first peak at around $1.6 \AA$ was attributed to four $\mathrm{N}$ atoms coordinating to the $\mathrm{Fe}$ (II) center. The second peak around $2.7 \AA$ was attributed to the $\mathrm{C}$ and $\mathrm{N}$ atoms, which were bound to the $\mathrm{N}$ atom in the pyrolic ring and the bridging $\mathrm{N}$ connecting the pyrolic rings, respectively.

The peak around $1.6 \AA$ in the RDF for G1AQ0.5Fe700 appeared; however, it was ill-defined. The peak around $2.7 \AA$ was small. The $\mathrm{Fe}-\mathrm{N}_{x}$ moiety generated only partially by the heat treatment at $700^{\circ} \mathrm{C}$. In the RDF for G1AQ0.5Fe800, the peak around $1.6 \AA$ similar to the first peak in the RDF for FePc and the peak around $2.7 \AA$

Table 1 Yields, specific surface areas $(S)$, and mean pore diameters $(d)$ of the carbon materials. The $-I_{\mathrm{K}}$ at the catalyst layers formed using the carbon materials is also listed.

\begin{tabular}{lcccc}
\hline & Yield $/ \%$ & $S / \mathrm{m}^{2} \mathrm{~g}^{-1}$ & $d / \mathrm{nm}$ & $-I_{\mathrm{K}} / \mathrm{A} \mathrm{g}^{-\mathbf{~}^{\mathrm{a}}}$ \\
\hline G1AQ0.5Fe700 & 50.2 & 9 & 8.78 & 0.01 \\
G1AQ0.5Fe800 & 40.1 & 253 & 2.21 & 0.43 \\
G1AQ0.5Fe900 & 19.5 & 686 & 2.54 & 1.78 \\
G1AQ0.5Fe1000 & 24.4 & 490 & 2.18 & 4.47 \\
G1AQ0.25Fe1000 & 3.9 & 1157 & 2.71 & 0.85 \\
G1AQ1Fe1000 & 29.0 & 368 & 2.29 & 0.01 \\
\hline
\end{tabular}

${ }^{\mathrm{a}} I_{\mathrm{K}}$ per mass of the carbon materials loaded on the rotating disk electrode at 0.7

$\mathrm{V}$ vs. a reversible hydrogen electrode as the reference electrode. 


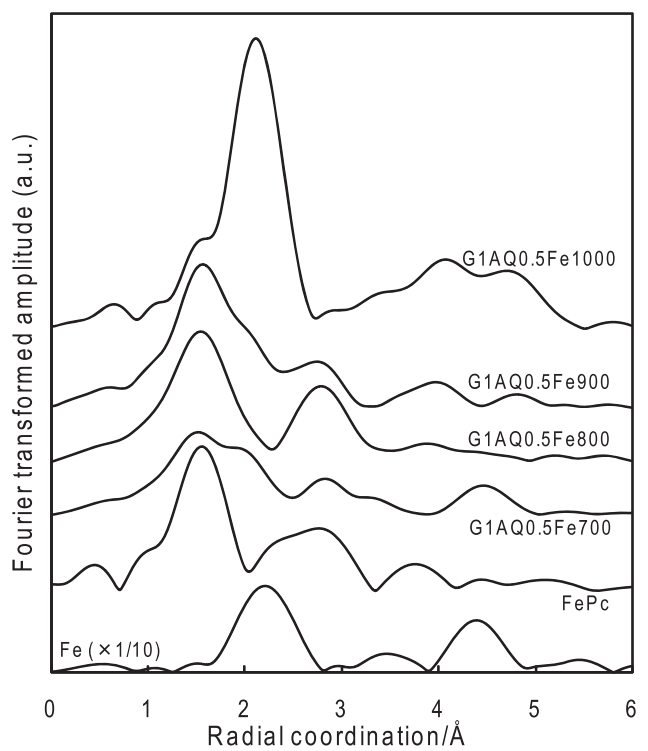

Fig. 1 RDFs calculated by Fourier transformation of extended X-ray adsorption fine structure spectra at the Fe $\mathrm{K}$-edge for the carbon materials, Fe foil, and FePc.

were clearly observed, indicating that the $\mathrm{Fe}-\mathrm{N}_{4}$ moiety was generated by the heat treatment at $800^{\circ} \mathrm{C}$. Although it is recognized that the identification of the nearest neighbor atom only from the EXAFS result is difficult, it might be reasonable to assume the presence of the $\mathrm{Fe}-\mathrm{N}_{4}$ moiety in the carbon materials, because DADCAQ is one of the raw materials that reacts with $\mathrm{Fe}$ to form FePc derivatives.

The first peak seen in Fe foil around $2.1 \AA$ slightly appeared in the RDF for G1AQ0.5Fe900 and dominated in that for G1AQ0.5Fe1000. The peak intensity around 2.7 $\AA$ decreased with an increase in the heat treatment temperature above $800^{\circ} \mathrm{C}$. The peak around $2.1 \AA$ indicated the generation of the $\mathrm{Fe}$ aggregates according to the RDF for Fe foil; nevertheless, the peaks around $1.6 \AA$ were still observed, which indicated the presence of the $\mathrm{Fe}-\mathrm{N}_{x}$ moiety.

These EXAFS results demonstrated that the high heat-treatment temperature was also necessary for the generation of the active site, although the Fe aggregates generated above $900^{\circ} \mathrm{C}$.

\subsection{Catalytic activity for oxygen reduction}

The catalytic activity of the carbon materials for the cathodic $\mathrm{O}_{2}$ reduction was examined by measuring the oxygen reduction current at the catalyst layer in $\mathrm{O}_{2}$-saturated $0.1 \mathrm{~mol} \mathrm{dm}{ }^{-3} \mathrm{HClO}_{4}$ at $25{ }^{\circ} \mathrm{C}$ using the GC RDEs. The $\mathrm{O}_{2}$ reduction current at the catalyst layer without the influence of mass transfer in the solution, $-I_{\mathrm{K}}$, was determined by the equation shown below.

$$
-\frac{1}{I}=-\frac{1}{I_{\mathrm{K}}}+\frac{1}{0.620 n F A D^{2 / 3} c v^{-1 / 6} \omega^{1 / 2}}
$$

where $I$ is the reduction current after subtracting the background current, $n$ is the number of electrons involved in the $\mathrm{O}_{2}$ reduction per molecule, $F$ is the Faraday constant, $A$ is the geometric area of the GC electrode, $D$ is the diffusion coefficient of $\mathrm{O}_{2}$ in the solution, $c$ is the concentration of $\mathrm{O}_{2}$ in the solution, $v$ is the kinematic viscosity of the solution, and $\omega$ is the angular frequency of the rotation. The sign of the cathodic current was taken as negative. The $I_{\mathrm{K}}$ values at $0.7 \mathrm{~V}$ vs. RHE as an indicator of the activity of the catalyst layer are listed in Table 1 . The $I_{\mathrm{K}}$ at $0.7 \mathrm{~V}$ was chosen based on the assumption that the kinetics could be largely reflected by the current at the potential without any experimental error caused by the low $\mathrm{O}_{2}$ reduction current at the higher potential.

The $-I_{\mathrm{K}}$ increased with an increase in the heat-treatment temperature. The increase in $-I_{K}$ from G1AQ0.5Fe700 to G1AQ0.5Fe800 and further to G1AQ0.5Fe900 were mainly due to the active site generation and the exposure of the active site caused by the pore development, respectively. The higher $-I_{\mathrm{K}}$ at G1AQ0.5Fe1000 than G1AQ0.5Fe900 in spite of the Fe aggregation and the less pore development was attributed to the improvement of the contact between the catalyst particles inside the catalyst layer. This attribution was based on the results of the cyclic voltammetry, showing the larger capacitive current for G1AQ0.5Fe1000 than G1AQ0.5Fe900.

The similar dependence of the $-I_{\mathrm{K}}$ on the degree of the pore development was observed for the carbon material formed from the mixture of the various glucose content. The increase in $-I_{\mathrm{K}}$ from G1AQ1Fe1000 to G1AQ0.5Fe1000 and the decrease to G1AQ0.25Fe1000 were due to the pore development and the loss of the contact between the catalyst particles, respectively. The previous study on activated carbon with various pore structures as a catalyst support for dispersed Pt also showed that the excessive pore development was disadvantageous for the contact between the catalyst particles inside the catalyst layer. ${ }^{6)}$

These results therefore indicated the necessity of the active site generation and the appropriate pore development to achieve the high activity. The carbon material formed in the highest heat-treatment temperature applied in this study and the optimized DADCAQ content, G1AQ0.5Fe1000, showed the highest $-I_{\mathrm{K}}$ value among the catalyst formed from the various glucose/nitrogen source/Fe salts, ${ }^{2-4)}$ although the activity should be further improved in future studies by examining the effects of the heat-treatment temperature above $1000^{\circ} \mathrm{C}$ and the glucose content change with constant Fe/DADCAQ ratio.

\section{Conclusion}

In the formation of the carbon material by the heat treatment of the mixture of glucose, DADCAQ, and Fe gluconate, the generation of $\mathrm{Fe}-\mathrm{N}_{x}$ moiety as the active site for cathodic $\mathrm{O}_{2}$ reduction was related to the heattreatment temperature. The pore development proceeded with an increase in the heat-treatment temperature up to $900{ }^{\circ} \mathrm{C}$ and a decrease in the DADCAQ content in the starting mixture. The high catalytic activity was achieved by the combination of the active site generation and the pore development without losing the electronic contact between the catalyst particles inside the 
catalyst layer.

\section{Acknowledgement}

The XAFS measurements were performed with the approval of SPring-8 (Proposal no. 2008A1891).

\section{References}

1) M. Lefèvre, E. Proietti, F. Jaouen, and J.-P. Dodelet, Science, 324, 71 (2009).

2) J. Maruyama and I. Abe, J. Electrochem. Soc., 154, B297
(2007)

3) J. Maruyama, N. Fukui, M. Kawaguchi, and I. Abe, J. Power Sources, 182, 489 (2008).

4) J. Maruyama, N. Fukui, M. Kawaguchi, and I. Abe, J. Power Sources, 194, 655 (2009).

5) J. Maruyama, N. Fukui, M. Kawaguchi, T. Hasegawa, H. Kawano, T. Fukuhara, and S. Iwasaki, Carbon, 48, 3271 (2010).

6) J. Maruyama, K.-i. Sumino, M. Kawaguchi, and I. Abe, Carbon, 42, 3115 (2004). 